OPEN ACCESS

Edited by:

Xuyu Zhou,

Chinese Academy of Sciences, China

Reviewed by:

Guoping Deng,

Peking University, China

Chuan Wu,

National Institutes of Health (NIH),

United States

*Correspondence:

Honglin Wang

honglin.wang@sjtu.edu.cn

Specialty section:

This article was submitted to

T Cell Biology,

a section of the journal

Frontiers in Immunology

Received: 30 September 2020

Accepted: 26 October 2020

Published: 20 November 2020

Citation:

Luo Y and Wang H (2020) Effects of

Non-Coding RNA on Regulatory

$T$ Cells and Implications for Treatment

of Immunological Diseases.

Front. Immunol. 11:612060.

doi: 10.3389/fimmu.2020.612060

\section{Effects of Non-Coding RNA on Regulatory T Cells and Implications for Treatment of Immunological Diseases}

\author{
Yuanhanyu Luo and Honglin Wang* \\ Institute of Translational Medicine, Shanghai General Hospital, Shanghai Jiao Tong University School of Medicine (SJTU-SM), \\ Shanghai, China
}

Regulatory T cells (Tregs) are essential for regulating immune reactions and maintaining immune homeostasis. Non-coding RNAs (ncRNAs), including microRNAs and long noncoding RNAs, usually do not encode proteins but regulate intracellular biological processes at post-transcriptional levels. These ncRNAs have been demonstrated as key post-transcriptional regulators in the commitment of Tregs lineage and the plasticity of Tregs function. These ncRNAs can further be manipulated to benefit human immunological disorders caused by Tregs dysfunction. This review summarizes the effects of ncRNAs on Tregs and their potentials to be targets or approaches for the treatment of immunological diseases involving Tregs.

Keywords: regulatory T cells, non-coding RNAs, post-transcriptional regulation, immunological tolerance, immunological disorders

\section{INTRODUCTION}

Regulatory $\mathrm{T}$ cells (Tregs), recognized as a specialized subset of $\mathrm{CD} 4^{+} \mathrm{T}$ cells, are essential mediators in maintaining immune tolerance by suppressing immune reactions (1). Three subsets of $\mathrm{CD} 4^{+}$ Tregs have been characterized, namely thymus-derived Treg (tTreg) and the peripherally induced Treg (pTreg) developed from mature $\mathrm{CD} 4^{+}$conventional $\mathrm{T}$ cells outside of the thymus and in vitroinduced regulatory T (iTreg) cells (2). Forkhead box P3 (Foxp3) has been identified as a master transcriptional factor in maintaining differentiation and suppressive function of Tregs (3). Downregulated Foxp3 expression commonly causes immune dysregulation, which elicits autoimmune diseases (4). Mutation in the Foxp3 gene impairs Tregs development and function, which further causes immune dysregulation polyendocrinopathy and enteropathy X-linked syndrome along with other grievous autoimmune diseases. Likewise, Foxp3-depleted or Foxp3mutated mice have Tregs deficiency and development of autoimmunity $(3,5,6)$.

Transcriptome and proteome studies of Tregs unveil post-transcriptional regulators manipulating Tregs development and function, which highlights the importance of non-coding RNA regulation (7). Non-coding RNAs (ncRNAs), comprising microRNA and long non-coding RNAs etc., are not able to 
encode proteins but still contain important information (8). MicroRNAs (miRNA) commonly contain $\sim 22$ nucleotides, which have function in RNA silencing and post-transcriptional regulation of gene expression. Their altered expression is observed in specific biological and pathological processes. MiRNAs bind to their respective target RNAs either in the translational regions or in the 3' untranslated regions (3'UTR). Particularly, miRNAs modifies mouse Tregs development, homeostasis, and normal function but in humans, their roles remain elusive (9). miRNAs play a crucial role in preserving Tregs suppressive function through Dicer-dependent pathway (10). LncRNAs harbor an abundant proportion of the noncoding transcriptomes. However, lncRNAs characteristics, function mechanisms in Tregs still remain obscure.

In this review, we firstly introduce the biogenesis of non-coding RNAs and exertion of suppressive function in Tregs. Then we summarize ncRNA-mediated post-transcriptional modifications of Tregs and discuss the potential therapeutic strategies for human immunological disorders with dysfunctional Tregs involved.

CD4+CD25+Foxp3+ regulatory T cells (Tregs) play a crucial role in maintaining immune homeostasis and self-tolerance through suppressing excess immune responses. Foxp3, the master transcription factor, is highly expressed in Tregs. Researchers found that Foxp3 programs development and function of Tregs since ectopic Foxp3 expression confers suppressor function in Tregs (11). Targeted deletion of mice Foxp3 resulted in severe lymphoproliferative autoimmune syndrome with evidently enlarged spleen and lymph nodes. Mutations of FOXP3 in human trigger several immune disorders, polyendocrinopathy, enteropathy and X-linked syndrome (IPEX) (12).

In vitro model systems identify the mechanisms by Tregs to suppress a large range of target cell types. These mechanisms can be divided into those that target responder Foxp $3^{-} \mathrm{T}$ cells and those that initially target antigen-presenting cells. Tregs secret multiple suppressive cytokines like IL-10, TGF- $\beta$ and IL-35 to regulate the activity of effector T cells. Tregs also overexpress CD25 (IL-2 receptor) to deprive local IL-2 resulting in the suppression of effector T cells. Activated Tregs may directly kill effector cells in a perforin-dependent and granzyme-mediated manner (13). On the other hand, Tregs may affect the function of APC indirectly inhibiting the activation of effector T cells. CTLA-4 on Tregs surface interacts with CD80 or CD86 on dendritic cells to downregulate costiulation, which is important for Tregs to exert their suppressive function. One other antigen on Tregs surface that affect Tregs function is LAG-3. LAG-3 binds to MHC class II molecules on immature DCs surface resulting in downregulated costimulatory capacity (14).

In vivo, Tregs residing in visceral adipose tissue (VAT) display distinct suppressive features in homeostasis maintenance (14). VAT-Tregs highly express the enzyme hydroxyprostaglandin dehydrogenase (HPGD), which converts $\mathrm{PGE}_{2}$ into the metabolite 15-keto $\mathrm{PGE}_{2}$. Once HPGD expression is promoted by PPAR $\gamma$, the generation of 15 -keto $\mathrm{PGE}_{2}$ inhibited other $\mathrm{T}$ cells activation and proliferation (14). Skeletal muscle Tregs and colonic Tregs also play an important part in local tissue homeostasis but their detailed mechanisms still need more research (14).

\section{BIOGENESIS OF NON-CODING RNAS TO REGULATE THE POST-TRANSCRIPTION MODIFICATION}

The RNA world is divided into two parts_potential and those without, also referred to as non-coding RNAs (ncRNAs). Though ncRNAs seem not to encode protein, they play an essential role in cell development and physiology. Also, the huge amount of ncRNAs form a complex regulation network in cells. ncRNA can be classified into two subclasses roughly by sequence size: small non-coding RNAs (ncRNAs smaller than 200 nucleotides) and long non-coding RNAs (lncRNAs, longer than 200 nt). Herein, we introduce biogenesis and mechanisms of microRNAs (miRNAs) and long non-coding RNAs in general $(15,16)$.

MicroRNAs (miRNAs) are commonly generated from transcriptional units or non-coding regions (17). In the beginning, miRNA genes are transcribed by RNA polymerase II into primary miRNA transcripts (pri-miRNA). pri-miRNA is relatively long with a 5'7-methyl guanosine cap and a 3' poly-adenylated hairpin structure (18). Pri-miRNA processing needs two ribonuclease III (RNase III) enzymes-D Drosha and Dicer. Firstly, in nucleus pri-miRNA transcripts are cleaved into 60-70 nucleotides precursor miRNA (pre-miRNA) by Drosha and its cofactor DiGeorge syndrome critical region gene 8 (DGCR8) forming a small hairpin structure $(19,20)$. Then pre-miRNAs are transported into the cytoplasm via Exportin 5 and be cut by another RNase III enzyme, Dicer into several small double-stranded RNA which is about 19-22 nucleotides long (21-23). One strand of the duplex is loaded into the RNA-induced silencing complex (RISC) while the other strand is degraded. RISC which consists of Dicer, TRBP, and the Argonaute proteins recognize target mRNAs in their 3'UTR to promote target mRNA degradation (24). According to such a unique mechanism, miRNA is able to specifically affect many key genes in the signal pathway.

Long non-coding RNAs (LncRNAs) are relatively less studied in ncRNA species due to their sequence heterogeneity. LncRNAs are transcribed by Pol II or Pol III and then $\operatorname{lncRNAs}$ are processed with m7G cap and polyadenylation (9). Subsequently, they are released into cytoplasm for further processing and stabilization to form a higher-order structure. LncRNAs exert their regulatory function through acting as sponges for miRNAs or degrading mRNAs in the cytoplasm. However, the detailed process of lncRNAs biogenesis and cofactors to ensure lncRNAs function remain elusive. It will be a challenging discovery for researchers to explore further (9).

\section{NON-CODING RNA MODIFICATIONS INVOLVED IN TREG LINEAGE COMMITMENT AND SUPPRESSIVE FUNCTION}

\section{MicroRNAs}

miRNAs play important regulatory roles in Tregs by pairing to target mRNAs. MiRNAs finely manage gene expression by directly interacting with 3'untranslated regions or other gene 
regions. MiRNAs form a complex network of regulation in Tregs (Figure 1).

miR15a/16, as studies showed in cord blood (CB) Tregs, were negatively associated with FOXP3 expression (25). miR15a/16 expression were significantly lower in CB Tregs when compared with conventional $\mathrm{T}$ cells. Overexpression of miR15a/16 in $\mathrm{CB}$ Tregs was demonstrated to inhibit normal FOXP3 transcription as well as CTLA4, a Foxp3-dependent gene in Tregs (25). On the other side, knockdown of miR15a/16 in CB T cells had been demonstrated to promote expression of FOXP3 and CTLA4, indicating that miR15a/16 play an important role in regulating Treg plasticity. miR15a/16 were reported to directly target specific regions of FOXP3 3'UTR. Thus, miR15a/16 are key regulators of Treg ratio at the post-transcriptional level (25).

miR-155 is highly expressed in Tregs. Genome-wide analysis reveals that Foxp3 bind to the B cell integration cluster (Bic) which harbors the primary miR-155 transcript $(26,27)$. Kohlhaas et al. reported that miR-155-deficient mice exhibited decreased numbers of Tregs in both thymi and spleens, although the suppressive function of Tregs in vitro remained unaffected (28). A high amount of miR-155 was maintained by Foxp3 expression in Tregs and sustained in a Foxp3-dependent pattern. miR-155 specifically targets suppressor of cytokine signaling 1 (SOCS1) to facilitate Tregs homeostasis. The reduced numbers of Tregs in miR155-deficient mice were in line with that in SOCS1-transgenic mice (29). miR-155 deletion in $\mathrm{CD}^{+}{ }^{+} \mathrm{T}$ cells compromised IL-2 production, which suggests that miR-155 likely regulates IL-2associated Tregs homeostasis via both cell-intrinsic and cellextrinsic mechanisms. Reduced STAT5 phosphorylation in miR155-deficient Tregs offered an alternative explanation for decreased Treg numbers in miR155-deficient mice (29).

miR-146 is prominently expressed in Tregs. Increased numbers of Foxp $3^{+}$Tregs in the periphery were observed in miR-146adeficient mice. Moreover, miR-146a-deficient Tregs were insufficient to restrain the activation of $\mathrm{T}$ effector cells (30). miR146 a deficiency lead to development and exhibition of a serious lymphoproliferative and myeloproliferative syndrome in mice at 6 months of age. Stat 1 is the target of miR-146a in human peripheral blood mononuclear cells (hPBMCs) since the 3' UTR of the human Stat1 gene perfectly matches the miR-146a sequence. Phosphorylated Stat1 is increased in miR-146a-deficient Tregs. Stat 1 is a key transcription factor downstream of IFN- $\gamma$ receptor signaling. miR-146a expression in Tregs is crucial to control Th1 immune responses mediated by IFN- $\boldsymbol{\gamma}$ via targeting Stat1 (30). Nevertheless, the role of miR-146a in autoimmune diseases remains to be determined using conditional miR-146a-deficient mice, since miR-146a-deficient myeloid cells promotes Tregs dysfunction and autoimmunity $(31,32)$.

miR-17-92 cluster is encoded by a single polycistronic host gene which produces 6 individual mature miRNAs. These miRNAs can be classified into 3 families based on sequence homology: the miR17 family (miR-17, miR-20, and miR-18a), the miR-19 family (miR$19 \mathrm{a}$ and miR-19b), and the miR-25 family (miR-92a). miR-17 and miR-19b inhibit iTreg differentiation. Consistently, elimination of the miR-17-92 cluster in $\mathrm{CD} 4{ }^{+} \mathrm{T}$ cells promoted Foxp3 expression. miR-17 directly targtes TGF- $\beta$ receptor II (Tgfbr 2 ) and cAMP- responsive element binding protein 1 (Creb1), both of which drive Treg differentiation (33). In addition, miR-17-92 engaged in costimulatory network of CD28, which is important for thymic development and survival of Tregs. CD28-mediated costimulation is crucial in the production of IL-10 in Tregs to ensure Treg immunosuppressive function. The frequency of IL-10 producing Tregs is remarkably reduced in miR-17-92 depleted Tregs demonstrating that miR-17-92's essential role in regulating Tregs normal function (34). Conclusively, miR-17-92 plays an important role in Tregs differentiation by interacting with certain important proteins like TGF- $\beta r$ and CREB1 and regulating IL-10 secretion in Tregs.

iTregs express high levels of miR-126. Disturbance of miR-126 using its antisense oligonucleotides (ASO) significantly inhibits the induction of Tregs. In vivo miR-126 abrogation dramatically downregulates the expression of Foxp3 in Tregs as well as CTLA4 and glucocorticoid induced tumor necrosis factor receptor (GITR) (35). miR-126 directly targets the 3'UTR of p85 $\beta$, which is an important regulatory subunit of PI3K involved in PI3K/Akt pathway (36). miR-126 ASO significantly elevate the expression of p85 $\beta$, contributing to the activation of PI3K/Akt pathway in Tregs, the reduced expression of Foxp3, and the impaired suppressive function of Tregs $(37,38)$.

miR-10a is a Treg-specific miRNA $(39,40)$. miR-10a gets involved in long-term maintenance of pTreg stability. Combination of alltrans Retinoid acid (ATRA) and TGF- $\beta$ dramatically boosts miR-10a in a dose-dependent manner, leading to optimal induction of miR10a in Tregs. miR-10a targets Bcl-6 and Ncor2 through binding to their 3'UTRs (41). In Peyer's patches (PP), Tregs can be converted to $\mathrm{T}$ follicular helper (Tfh) cells, which is limited by miR-10a via dampening Bcl-6 expression (41). Intriguingly, miR-10a is dispensable for the direct regulation of Foxp3 or other related factors in iTregs. In mice deficient of miR-10a, neither the number nor function of Tregs was altered, when treated with RA and TGF$\beta(41)$.

miR-142-3p regulates Tregs function in a unique way. Naturally, Tregs maintain high levels of cAMP and transfer cAMP to conventional $\mathrm{T}$ cells through gap junction channels to facilitate immunomodulation. Intracellular adenylyl cyclases (ACs) are key enzymes for cAMP generation. miR-142-3p directly targets the 3'UTR of AC9 in Tregs. Therefore, miR-142-3p restricts the generation of cAMP and impairs the suppressive function of Tregs. Blockage of miR-142-3p with inhibitors restores the cytokine production and suppressive function of Tregs. Foxp3 downregulates miR-142-3p to maintain the activity of AC9 and the production of cAMP. The downregulation of miR-142-3p might get involved in the mechanisms of which Foxp3 upregulates the expression of Treg-associated genes in the Tregs (42).

miR-125a is downregulated in peripheral $\mathrm{CD} 4^{+} \mathrm{T}$ cells of several human auto-inflammatory diseases including systemic lupus erythematosus and Crohn's disease as well as relevant mouse models (43). In miR-125a knockout mice, proportions of $\mathrm{CD}^{+}$ and $\mathrm{CD}^{+} \mathrm{T}$ cells in lymph nodes and spleens were not affected while the percentages and absolute numbers of Tregs suffered a significant decline (43). miR-125a deficiency impaired the ability of $\mathrm{T}$ cells to differentiate into Tregs in vitro. Mechanistically, miR-125a 


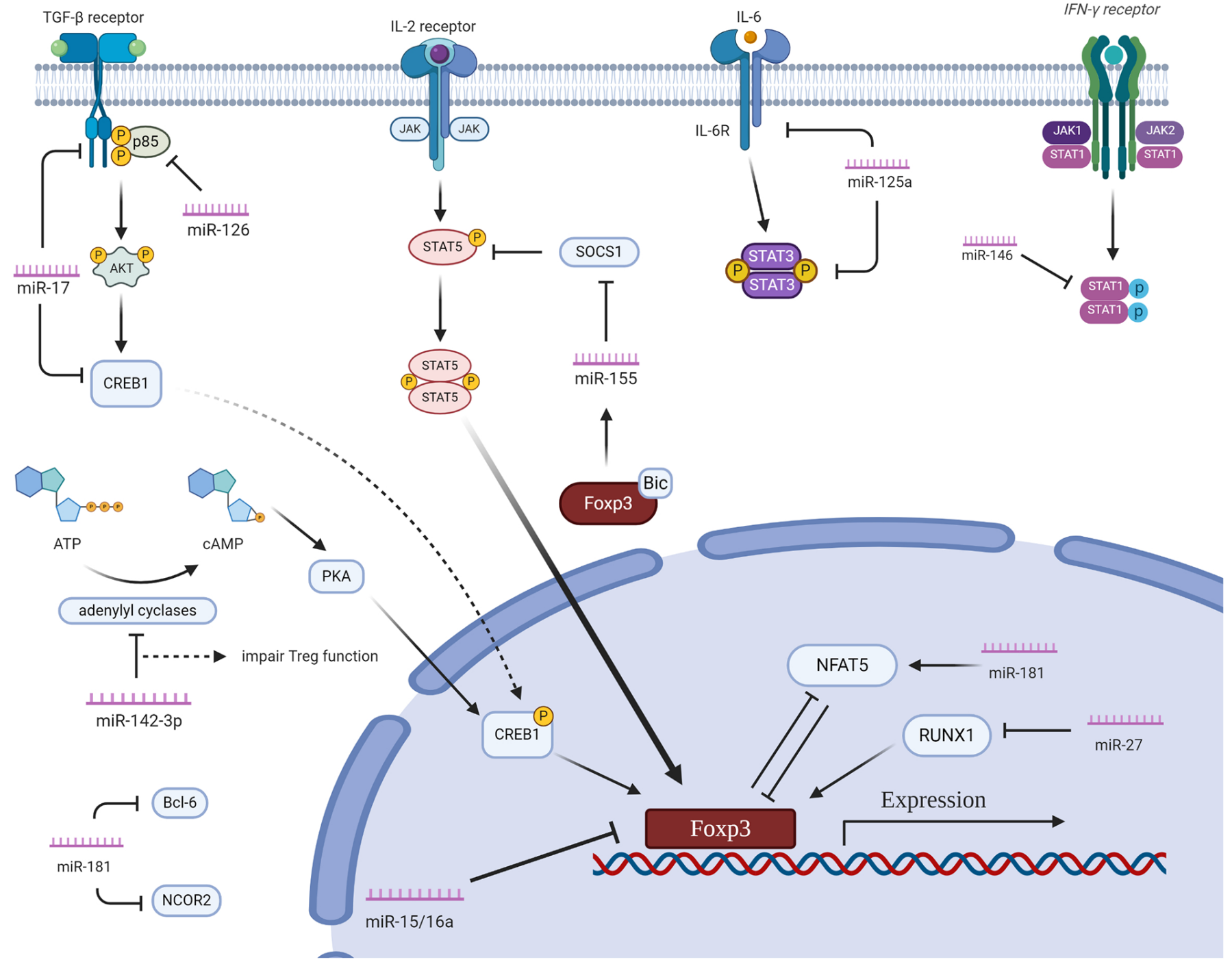

FIGURE 1 | The regulatory network of miRNAs in Tregs. miR15a/16 directly binds FOXP3, which inhibits Treg differentiation. miR-155 compromises IL-2 production via specifically targeting SOCS1. miR-146a downregulates Stat 1 to alleviate IFN- $\gamma$ signaling. miR-17 directly targtes TGF- $\beta$ receptor II and cAMP-responsive element binding protein 1 thus negatively regulating Treg differentiation. miR-126 targets the 3 'UTR of p85 $\beta$, an important regulatory subunit of PI3K, reducing the expression of Foxp3. miR-10a directly targets Bcl-6 and Ncor2. miR-142-3p binds with adenylyl cyclases, which impairs the suppressive effect of Tregs. miR-125a regulate the function of Tregs by targeting Stat3. miR-181a directly inhibits NFAT5 which is an important transcriptional factor for Foxp3. miR-27 downregulates RUNX1, which impairs the differentiation of Tregs. Created with BioRender.com. 
directly represses several targets which inhibit Tregs differentiation, including Stat3, IL-13, IFN- $\gamma$ and IL-6R (44). miR-125a also directly modulates CCR2 expression of Tregs in pancreatic draining lymph nodes, which limits Tregs migration and affects maintenance of immune tolerance in the pancreas (45).

Expression levels of IL-10 and TGF- $\beta$ are higher in miR-181aoverexpressing $\mathrm{CD} 4^{+} \mathrm{T}$ cells than controls. Although miR-181 does not influence Treg differentiation, it elevates IL-10 and TGF- $\beta$ via PI3K/Akt and SIRT1 pathways to enhance the suppressive function of Tregs (46). On the other hand, another study showed that enhancement of miR-181 up-regulated nuclear factor of activated T cells 5 (NFAT5), restraining Foxp $3^{+}$Tregs induction in vitro. This mechanism indicates that inhibited production of miR-181 promotes Tregs induction in mouse autoimmune model and alleviates murine islet autoimmunity in vivo (47). These results showed that miR-181a may play pro-inflammatory or antiinflammatory roles. Also, specific targeting miR-181a sheds light on medicine development aiming at limiting islet autoimmunity or other autoimmune diseases (47).

miR-27 belongs to the miR-23 27 24 family and is upregulated in T cells isolated from patients with multiple sclerosis (MS) (48). miR-27 directly repressed B lymphoma Mo-MLV insertion region 1 homolog (Bmi-1), whose protein stabilizes the Th2 transcription factor, GATA3. Thus, forced miR-27 overexpression represses Bmi1, facilitates GATA3 degradation and reduces Th2 responses. It triggers pathogenic Th1 responses in mice with $\mathrm{T}$ cell miR-27 overexpression (49). On the other hand, Forced miR-27 overexpression in murine $\mathrm{T}$ cells severely impaired Tregs generation by directly targeting runt-related transcription factor 1 (RUNX1), SMAD2/3, and c-Rel, which are known as members of the NF- $\mathrm{BB}$ family and play indispensable roles in initiating Foxp3 transcription. In addition, miR-27 overexpression suppresses Tregs function via targeting IL-10 (50) (Table 1).

\section{LncRNAs}

LncRNAs are commonly described to be longer than 200 nucleotides with the ability to modulate chromatin structure and regulate gene expression at both transcriptional and posttranscriptional levels.

Hepatocellular carcinoma up-regulated lncRNA (HULC) contributes to Tregs differentiation by targeting p18 and is engaged in hepatocellular carcinoma (HCC) development and progression (51).
lncRNA SNHG1 promotes Tregs differentiation and drives the progression of colorectal carcinoma. SNHG1 binds to miR-448, the latter directly targets Indoleamine 2,3-dioxygenase (IDO), which is a inducer of Tregs. Thus, SNHG1 induces Tregs differentiation and facilitates immune escape through suppressing miR-448 function $(52,53)$

Foxp3 long intergenic noncoding RNA (Flicr) impairs Tregs differentiation via reducing the chromatin accessibility in the Foxp3 locus under low IL-2 levels. Once Flicr is deleted, Treg restores stabilized level of Foxp3 levels, suggesting that Flicr modulation is likely to serve as a switch to enhance or inhibit Treg suppressive function (54).

\section{IMPLICATIONS FOR THE TREATMENT OF HUMAN IMMUNOLOGICAL DISEASES}

Kawasaki disease (KD) is an acute systemic inflammatory disease, which subjects children to coronary artery aneurysms, myocardial infarction or even sudden death (55). KD onset involves immune dysfunction with descending Treg proportions and decreased expression of Foxp3. As previously mentioned, miR-155 is required for normal Tregs frequency and function. KD patients show decreased miR-155, which is a factor contributing to Tregs deficiency and KD progression (56). Restoring miR-155 expression in $\mathrm{KD}$ patients is a promising strategy.

MiR-21 has been demonstrated to play an important role in regulating the Th17/Treg balance. miR-21 upregulates Foxp3 and promotes Tregs differentiation. miR-21 alleviates rheumatoid arthritis (RA) via boosting the immunomodulatory properties of Tregs (57).

miR-31 was found to directly target Foxp3 and lentiviral transduction of miR-31 evidently reduced Foxp3 expression (8). In another study, miR-31 deficiency boosted Tregs differentiation through targeting protein phosphatase $6 \mathrm{c}$ (Ppp6c), which then led to decreased blood pressure, relieved vascular damage and alleviated hypertension pathology. Studies also showed that miR31 inhibited pTreg generation via directly targeting Gprc5a (58).

miR-210 has been revealed to be critical for psoriasis pathology in several aspects (59). miR-210 expression is upregulated in peripheral blood $\mathrm{CD}^{+} \mathrm{T}$ cells as well as in the skin lesions of patients with psoriasis. Functionally, overexpression of miR-210 aggravates psoriasis and deletion of miR-210 inhibits psoriasis

TABLE 1 | Characteristics of MiRNAs in Tregs.

\begin{tabular}{|c|c|c|c|}
\hline MiRNAs & Impact on Tregs & Targets & Human immunological diseases or animal models \\
\hline $\operatorname{miR} 15 a / 16$ & Impair suppressive function & Foxp3 & Human cord blood \\
\hline $\operatorname{miR}-155$ & Maintain suppressive function & SOCS1 & Mice and Kawasaki diseases \\
\hline $\mathrm{miR}-146$ & Promote suppressive function & IFN- $\gamma$ signaling & Mice \\
\hline miR-17-92 & Promote Tregs differentiation & TGF- $\beta$ signaling pathway & Mice \\
\hline miR-126 & Promote suppressive function & PI3K/Akt pathway & Mice \\
\hline $\mathrm{miR}-10 \mathrm{a}$ & Maintain Tregs stability & Bcl-6 and Ncor2 & Mice \\
\hline $\operatorname{miR}-142-3 p$ & Maintain Tregs suppressive function & Adenylyl cyclases & Mice \\
\hline miR-125a & Inhibit Tregs differentiation & Stat3, IL-13 & Lupus erythematosus and Crohn's disease as well as mice \\
\hline miR-181a & Enhance the suppressive function & NFAT5 & Islet autoimmunity in mice \\
\hline $\operatorname{miR}-27$ & Impair Tregs generation & RUNX1, SMAD2/3 and c-Rel & Human multiple sclerosis \\
\hline
\end{tabular}


development. Thus, $\mathrm{miR}-210$ proves a promising drug target for the treatment of psoriais (60).

miR-214, conventionally considered as an oncogenic miRNA, promotes human ovarian cancer through directly targeting phosphatase and tensin homolog (PTEN). Secreted miR-214 from Lewis lung carcinoma (LLC) cells is a strong inducer of Tregs expansion both in vitro and in vivo. Consistently, blockage of miR214 using ASO undermines Tregs induction and thus inhibits tumor growth. Importantly, cell-derived microvesicles (MVs) were employed to deliver miR-214 ASO to human peripheral $\mathrm{CD}^{+} \mathrm{T}$ cells, which increases PTEN expression and inhibits Tregs expansion. This may offer an innovative avenue to tumor therapy (61).

\section{CONCLUSION}

Non-coding RNAs play an important role in regulating Tregs differentiation and function. We conclude a range of miRNAs and long-coding RNAs and reveal their effects on Tregs. These miRNAs provide a pool of targets for treatments of Tregsmediated autoimmune disease. However, this field needs more

\section{REFERENCES}

1. Sakaguchi S, Sakaguchi N, Asano M, Itoh MMT. Immunologic Self-Tolerance Maintained by Activated T Cells Expressing 11-2 Receptor a-Chains (CD25).Breakdown of a Single Mechanism of Self-Tolerance Causes Various Autoimmune Diseases. J Immunol (1995) 1151-1164):155.

2. Sakaguchi S. Naturally arising CD4+ regulatory T cells for immunologic selftolerance and negative control of immune responses. Annu Rev Immunol (2004) 22:531-62. doi: 10.1146/annurev.immunol.21.120601.141122

3. Fontenot JD, Gavin MA, Rudensky AY. Foxp3 programs the development and function of CD4+CD25+ regulatory T cells. Nat Immunol (2003) 4(4):330-6. doi: $10.1038 /$ ni904

4. Gavin MA, Torgerson TR, Houston E, DeRoos P, Ho WY, Stray-Pedersen A, et al. Single-cell analysis of normal and FOXP3-mutant human T cells: FOXP3 expression without regulatory T cell development. Proc Natl Acad Sci (2006) 103(17):6659-64. doi: 10.1073/PNAS.0509484103

5. Wan YY, Flavell RA. Regulatory T-cell functions are subverted and converted owing to attenuated Foxp3 expression. Nature (2007) 445(7129):766-70. doi: 10.1038/ nature 05479

6. Hori S, Nomura T, Sakaguchi S. Control of regulatory T cell development by the transcription factor Foxp3. Science (2003) 299:1057-61. doi: 10.1126/science.1079490

7. Mattick JS, Makunin IV. Non-coding RNA. Hum Mol Genet (2006) 15 Spec No(1):17-29. doi: 10.1093/hmg/ddl046

8. Rouas R, Fayyad-Kazan H, El Zien N, Lewalle P, Rothé F, Simion A, et al. Human natural Treg microRNA signature: Role of microRNA-31 and microRNA-21 in FOXP3 expression. Eur J Immunol (2009) 39(6):1608-18. doi: 10.1002/eji.200838509

9. Beermann J, Piccoli MT, Viereck J, Thum T. Non-coding rnas in development and disease: Background, mechanisms, and therapeutic approaches. Physiol Rev (2016) 96(4):1297-325. doi: 10.1152/physrev.00041.2015

10. Liston A, Lu L, Carroll DO, Tarakhovsky A, Rudensky AY. Dicer-dependent microRNA pathway safeguards regulatory T cell function. J Exp Med (2008) 205(9):1993-2004. doi: 10.1084/jem.20081062

11. Grossman WJ, Verbsky JW, Tollefsen BL, Kemper C, Atkinson JP, Ley TJ. Differential expression of granzymes A and B in human cytotoxic lymphocyte subsets and T regulatory cells. Blood (2004) 104(9):2840-8. doi: 10.1182/ blood-2004-03-0859

12. Bennett CL, Christie J, Ramsdell F, Brunkow ME, Ferguson PJ, Whitesell L, et al. The immune dysregulation, polyendocrinopathy, enteropathy, X-linked exploration. The roles of several miRNA in Tregs and other $\mathrm{T}$ cell subsets are still elusive both in vitro and in vivo. Therefore, what we need is the big picture of interaction between miRNAs in Tregs, which will be helpful for understanding underlying mechanisms and discovering potential therapies.

\section{AUTHOR CONTRIBUTIONS}

YL wrote the manuscript. HW and YL designed the structure and content of this review. All authors contributed to the article and approved the submitted version.

\section{FUNDING}

This work is supported by grants from the National Natural Science Foundation of China $(81930088,81725018,81703118$ and 81803123), Shanghai Collaborative Innovation Center for Translational Medicine (TM201925), Innovative Research Team of High-Level Local Universities in Shanghai.

syndrome (IPEX) is caused by mutations of FOXP3. Nat Genet (2001) 27 (1):20-1. doi: $10.1038 / 83713$

13. Shevach EM. Mechanisms of Foxp3+ T Regulatory Cell-Mediated Suppression. Immunity (2009) 30(5):636-45. doi: 10.1016/j.immuni.2009. 04.010

14. Schmidleithner L, Thabet Y, Schönfeld E, Köhne M, Sommer D, Abdullah Z, et al. Enzymatic Activity of HPGD in Treg Cells Suppresses Tconv Cells to Maintain Adipose Tissue Homeostasis and Prevent Metabolic Dysfunction. Immunity (2019) 50(5):1232-48.e14. doi: 10.1016/j.immuni.2019.03.014

15. Lee Y, Jeon K, Lee JT, Kim S, Kim VN. MicroRNA maturation: Stepwise processing and subcellular localization. EMBO J (2002) 21(17):4663-70. doi: 10.1093/emboj/cdf476

16. Rodriguez A, Griffiths-Jones S, Ashurst JL, Bradley A. Identification of mammalian microRNA host genes and transcription units. Genome Res (2004) 14(10 A):1902-10. doi: 10.1101/gr.2722704

17. Denli AM, Tops BBJ, Plasterk RHA, Ketting RF, Hannon GJ. Processing of primary microRNAs by the Microprocessor complex. Nature (2004) 432 (7014):231-5. doi: 10.1038/nature03049

18. Gregory RI, Yan KP, Amuthan G, Chendrimada T, Doratotaj B, Cooch N, et al. The Microprocessor complex mediates the genesis of microRNAs. Nature (2004) 432(7014):235-40. doi: 10.1038/nature03120

19. Lund E, Güttinger S, Calado A, Dahlberg JE, Kutay U. Nuclear Export of MicroRNA Precursors. Science (2004) 303(5654):95-8. doi: 10.1126/science.1090599

20. Zhang H, Kolb FA, Jaskiewicz L, Westhof E, Filipowicz W. Single processing center models for human Dicer and bacterial RNase III. Cell (2004) 118(1):5768. doi: 10.1016/j.cell.2004.06.017

21. Kawamata T, Tomari Y. Making RISC. Trends Biochem Sci (2010) 35(7):36876. doi: 10.1016/j.tibs.2010.03.009

22. Ameres SL, Martinez J, Schroeder R. Molecular Basis for Target RNA Recognition and Cleavage by Human RISC. Cell (2007) 130(1):101-12. doi: 10.1016/j.cell.2007.04.037

23. Bartel DP. MicroRNAs: Target Recognition and Regulatory Functions. Cell (2009) 136(2):215-33. doi: 10.1016/j.cell.2009.01.002

24. Kapranov P, Cheng J, Dike S, Nix DA, Duttagupta R, Willingham AT, et al. RNA maps reveal new RNA classes and a possible function for pervasive transcription. Science (2007) 316(5830):1484-8. doi: 10.1126/science.1138341

25. Liu X, Robinson SN, Setoyama T, Tung SS, D'Abundo L, Shah MY, et al. FOXP3 is a direct target of miR15a/16 in umbilical cord blood regulatory $\mathrm{T}$ cells. Bone Marrow Transplant (2014) 49(6):793-9. doi: 10.1038/bmt.2014.57 
26. Zheng Y, Josefowicz SZ, Kas A, Chu TT, Gavin MA, Rudensky AY. Genomewide analysis of Foxp3 target genes in developing and mature regulatory $\mathrm{T}$ cells. Nature (2007) 445(7130):936-40. doi: 10.1038/nature05563

27. Marson A, Kretschmer K, Frampton GM, Jacobsen ES, Polansky JK, MacIsaac $\mathrm{KD}$, et al. Foxp3 occupancy and regulation of key target genes during T-cell stimulation. Nature (2007) 445(7130):931-5. doi: 10.1038/nature05478

28. Kohlhaas S, Garden OA, Scudamore C, Turner M, Okkenhaug K, Vigorito E. Cutting Edge: The Foxp3 Target miR-155 Contributes to the Development of Regulatory T Cells. J Immunol (2009) 182(5):2578-82. doi: 10.4049/ jimmunol.0803162

29. Lu LF, Thai TH, Calado DP, Chaudhry A, Kubo M, Tanaka K, et al. Foxp3Dependent MicroRNA155 Confers Competitive Fitness to Regulatory T Cells by Targeting SOCS1 Protein. Immunity (2009) 30(1):80-91. doi: 10.1016/ j.immuni.2008.11.010

30. Lu LF, Boldin MP, Chaudhry A, Lin LL, Taganov KD, Hanada T, et al. Function of miR-146a in Controlling Treg Cell-Mediated Regulation of Th1 Responses. Cell (2010) 142(6):914-29. doi: 10.1016/j.cell.2010.08.012

31. Zhao JL, Rao DS, Boldin MP, Taganov KD, O'Connell RM, Baltimore D. NF$\kappa \mathrm{B}$ dysregulation in microRNA-146a-deficient mice drives the development of myeloid malignancies. Proc Natl Acad Sci U S A (2011) 108(22):9184-9. doi: 10.1073/pnas.1105398108

32. Boldin MP, Taganov KD, Rao DS, Yang L, Zhao JL, Kalwani M, et al. miR146a is a significant brake on autoimmunity, myeloproliferation, and cancer in mice. J Exp Med (2011) 208(6):1189-201. doi: 10.1084/jem.20101823

33. Jiang S, Li C, Olive V, Lykken E, Feng F, Sevilla J, et al. Molecular dissection of the miR-17-92 cluster's critical dual roles in promoting Th1 responses and preventing inducible Treg differentiation. Blood (2011) 118(20):5487-97. doi: 10.1182/blood-2011-05-355644

34. de Kouchkovsky D, Esensten JH, Rosenthal WL, Morar MM, Bluestone JA, Jeker LT. microRNA-17-92 Regulates IL-10 Production by Regulatory T Cells and Control of Experimental Autoimmune Encephalomyelitis. J Immunol (2013) 191(4):1594-605. doi: 10.4049/jimmunol.1203567

35. Qin A, Wen Z, Zhou Y, Li Y, Li Y, Luo J, et al. MicroRNA-126 regulates the induction and function of CD4+ Foxp3+ regulatory T cells through PI3K/ AKT pathway. J Cell Mol Med (2013) 17(2):252-64. doi: 10.1111/jcmm.12003

36. Guo C, Sah JF, Beard L, Willson JKV, Markowitz SD, Guda K. The noncoding RNA, miR-126, suppresses the growth of neoplastic cells by targeting phosphatidylinositol 3-kinase signaling and is frequently lost in colon cancers. Genes Chromosomes Cancer (2008) 47(11):939-46. doi: 10.1002/gcc.20596

37. Crellin NK, Garcia RV, Levings MK. Altered activation of AKT is required for the suppressive function of human CD4+CD25+ T regulatory cells. Blood (2007) 109(5):2014-22. doi: 10.1182/blood-2006-07-035279

38. Merkenschlager M, von Boehmer H. PI3 kinase signalling blocks Foxp3 expression by sequestering Foxo factors. J Exp Med (2010) 207(7):1347-50. doi: 10.1084/jem.20101156

39. Kuchen S, Resch W, Yamane A, Kuo N, Li Z, Chakraborty T, et al. Regulation of MicroRNA expression and abundance during lymphopoiesis. Immunity (2010) 32(6):828-39. doi: 10.1016/j.immuni.2010.05.009

40. Jeker LT, Zhou X, Gershberg K, de Kouchkovsky D, Morar MM, Stadthagen G, et al. MicroRNA 10a marks regulatory T cells. PloS One (2012) 7(5):1-8. doi: 10.1371/journal.pone.0036684

41. Takahashi H, Kanno T, Nakayamada S, Hirahara K, Sciumé G, Muljo SA, et al. TGF- $\beta$ and retinoic acid induce miR-10a, which targets Bcl- 6 and constrains helper T cell plasticity. Nat Immunol (2013) 13(6):587-95. doi: 10.1038/ ni.2286.TGF-

42. Huang B, Zhao J, Lei Z, Shen S, Li D, Shen GX, et al. miR-142-3p restricts cAMP production in CD4+CD25- T cells and CD4+CD25+ TREG cells by targeting AC9 mRNA. EMBO Rep (2009) 10(2):180-5. doi: 10.1038/ embor.2008.224

43. Pan W, Zhu S, Dai D, Liu Z, Li D, Li B, et al. MiR-125a targets effector programs to stabilize Treg-mediated immune homeostasis. Nat Commun (2015) 6(2014):1-12. doi: 10.1038/ncomms8096

44. Li D, Kong C, Tsun A, Chen C, Song H, Shi G, et al. MiR-125a-5p Decreases the Sensitivity of Treg cells Toward IL-6-Mediated Conversion by Inhibiting IL-6R and STAT3 Expression. Sci Rep (2015) 5:1-10. doi: 10.1038/srep14615

45. Sebastiani G, Ventriglia G, Stabilini A, Socci C, Morsiani C, Laurenzi A, et al. Regulatory T-cells from pancreatic lymphnodes of patients with type-1 diabetes express increased levels of microRNA MIR-125a-5p that limits CCR2 expression. Sci Rep (2017) 7(1):1-9. doi: 10.1038/s41598-017-07172-1

46. Zeng Q, Liu W, Luo R, Lu G. MicroRNA-181a and microRNA-155 are involved in the regulation of the differentiation and function of regulatory $\mathrm{T}$ cells in allergic rhinitis children. Pediatr Allergy Immunol (2019) 30(4):43442. doi: $10.1111 /$ pai. 13038

47. Serr I, Scherm MG, Zahm AM, Schug J, Flynn VK, Hippich M, et al. A miRNA181a/NFAT5 axis links impaired T cell tolerance induction with autoimmune type 1 diabetes. Sci Trans Med (2018) 10(422):1-15. doi: 10.1126/scitranslmed.aag1782

48. Cho S, Wu CJ, Yasuda T, Cruz LO, Khan AA, Lin LL, et al. miR-23 27 24 clusters control effector T cell differentiation and function. J Exp Med (2016) 213(2):235-49. doi: 10.1084/jem.20150990

49. Guerau-De-Arellano M, Smith KM, Godlewski J, Liu Y, Winger R, Lawler SE, et al. Micro-RNA dysregulation in multiple sclerosis favours proinflammatory T-cell-mediated autoimmunity. Brain (2011) 134(12):357586. doi: 10.1093/brain/awr262

50. Cruz LO, Hashemifar SS, Wu CJ, Cho S, Nguyen DT, Lin LL, et al. Excessive expression of MIR-27 impairs Treg-mediated immunological tolerance. J Clin Inves (2017) 127(2):530-42. doi: 10.1172/JCI88415

51. Zhao J, Fan Y, Wang K, Ni X, Gu J, Lu H, et al. LncRNA HULC affects the differentiation of Treg in HBV-related liver cirrhosis. Int Immunopharmacol (2015) 28(2):901-5. doi: 10.1016/j.intimp.2015.04.028

52. Sun X, Wang Z, Yuan W. Down-regulated long non-coding RNA SNHG1 inhibits tumor genesis of colorectal carcinoma. Cancer Biomarkers (2017) 20 (1):67-73. doi: 10.3233/CBM-170112

53. Pei X, Wang X, Li H. LncRNA SNHG1 regulates the differentiation of Treg cells and affects the immune escape of breast cancer via regulating miR-448/ IDO. Int J Biol Macromol (2018) 118(2017):24-30. doi: 10.1016/ j.ijbiomac.2018.06.033

54. Zemmour D, Pratama A, Loughhead SM, Mathis D, Benoist C. Flicr, a long noncoding RNA, modulates Foxp3 expression and autoimmunity. Proc Natl Acad Sci U S A (2017) 114(17):E3472-80. doi: 10.1073/pnas.1700946114

55. Rowley AH, Shulman ST. Pathogenesis and management of Kawasaki disease. Expert Rev Anti-Infect Ther (2010) 8(2):197-203. doi: 10.1586/eri.09.109

56. Jia S, Li C, Wang G, Yang J, Zu Y. The T helper type 17/regulatory T cell imbalance in patients with acute Kawasaki disease. Clin Exp Immunol (2010) 162(1):131-7. doi: 10.1111/j.1365-2249.2010.04236.x

57. Jin S, Chen H, Li Y, Zhong H, Sun W, Wang J, et al. Maresin 1 improves the Treg/Th17 imbalance in rheumatoid arthritis through miR-21. Ann Rheumatic Dis (2018) 77(11):1644-52. doi: 10.1136/annrheumdis-2018213511

58. Zhang L, Ke F, Liu Z, Bai J, Liu J, Yan S, et al. MicroRNA-31 negatively regulates peripherally derived regulatory $\mathrm{T}$-cell generation by repressing retinoic acid-inducible protein 3. Nat Commun (2015) 6:7639. doi: 10.1038/ ncomms8639

59. Deng Y, Chang C, Lu Q. The Inflammatory Response in Psoriasis: a Comprehensive Review. Clin Rev Allergy Immunol (2016) 50(3):377-89. doi: 10.1007/s12016-016-8535-x

60. Wu R, Zeng J, Yuan J, Deng X, Huang Y, Chen L, et al. MicroRNA-210 overexpression promotes psoriasis-like inflammation by inducing Th1 and Th17 cell differentiation. J Clin Inves (2018) 128(6):2551-68. doi: 10.1172/ JCI97426

61. Yin Y, Cai X, Chen X, Liang H, Zhang Y, Li J, et al. Tumor-secreted miR-214 induces regulatory T cells: A major link between immune evasion and tumor growth. Cell Res (2014) 24(10):1164-80. doi: 10.1038/cr.2014.121

Conflict of Interest: The authors declare that the research was conducted in the absence of any commercial or financial relationships that could be construed as a potential conflict of interest.

Copyright (c) 2020 Luo and Wang. This is an open-access article distributed under the terms of the Creative Commons Attribution License (CC BY). The use, distribution or reproduction in other forums is permitted, provided the original author(s) and the copyright owner(s) are credited and that the original publication in this journal is cited, in accordance with accepted academic practice. No use, distribution or reproduction is permitted which does not comply with these terms. 